\title{
Instrumentos de Medição da Qualidade de Vida em Idade Pediátrica em Cuidados Paliativos
}

\author{
Measuring Instruments of the Quality of Life Pediatric Palliative Care
}

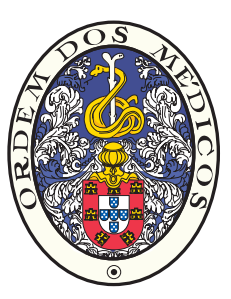

\author{
Joana ARAÚJO ${ }^{1,2}$, Marília DOURADO $\bigotimes^{3}$, Pedro Lopes FERREIRA ${ }^{4}$ \\ Acta Med Port 2015 Jul-Aug;28(4):501-512
}

\section{RESUMO}

Introdução: Os cuidados paliativos estão intimamente ligados ao conceito de qualidade de vida. Neste trabalho, focaremos a nossa atenção na necessidade de avaliar a qualidade de vida em cuidados paliativos pediátricos oncológicos.

Objetivo: Descrever/comparar os instrumentos de medição da qualidade de vida em cuidados paliativos pediátricos oncológicos.

Material e Métodos: Pesquisa da bibliográfica dos vários instrumentos de medição da qualidade de vida das crianças em cuidados paliativos, em inglês e português, entre 2000 e 2013, nas bases de dados reconhecidas para este efeito.

Resultados: Encontraram-se quinze instrumentos de medição: 10 genéricos e cinco específicos. Identificou-se, para cada instrumento de medição, país de origem, faixa etária para que se encontra direcionado, modalidade de preenchimento, número de dimensões avaliadas, descrição das dimensões, número de perguntas, propriedades psicométricas e validação para português.

Discussão: Verifica-se que tem havido uma crescente preocupação em medir a qualidade de vida em idade pediátrica e que a maioria dos instrumentos de medição foi desenhada, nos Estados Unidos, após 1994, coincidindo temporalmente com a definição de qualidade de vida da Organização Mundial de Saúde. Relativamente à faixa etária, a maioria dos instrumentos foi desenvolvida para crianças com idade igual ou superior a oito anos não existindo nenhum para ser respondido apenas pela criança. Podemos verificar que a maioria dos instrumentos de medição, sobretudo os mais atuais, procuram envolver a criança na avaliação da sua própria qualidade de vida relacionada com a saúde através do autopreenchimento $(n=10)$. Porém, há ainda uma dependência substancial dos pais para a medição da qualidade de vida relacionada com a saúde dos seus filhos, apesar dos estudos que demonstram diferenças na perceção de qualidade de vida relacionada com a saúde entre a criança e os pais. Mas como muitas crianças não têm capacidade de fornecer dados sobre a sua qualidade de vida relacionada com a saúde, quer devido à idade quer porque estão doentes ou com incapacidade funcional, a única possibilidade de obter informação sobre a qualidade de vida relacionada com a saúde destas crianças é o recurso aos pais, a quem é pedido que reflitam sobre a perceção da sua criança, ou adolescente, podendo, assim, justificar-se o preenchimento integral pelos pais de alguns instrumentos de medição. Os instrumentos de medição de qualidade de vida relacionada com a saúde em pediatria são geralmente multidimensionais, destinados a medir o ponto de vista subjetivo em relação ao impacto que a doença e o tratamento têm sobre o bem-estar físico, psicológico e social. Assim, a grande variedade de dimensões, as diferenças no número de dimensões e no número de perguntas entre os diversos instrumentos tendem a refletir os diferentes estádios de desenvolvimento psicomotor da população-alvo.

Conclusão: As dimensões mais comummente medidas foram a capacidade física, impacto emocional/psicológico, impacto social e na escola, seguidas da dor e desconforto e nível de atividade. A maioria dos instrumentos é desenvolvida para crianças com idade igual/superior a oito anos. Grande parte dos questionários é de autopreenchimento. Outros podem ser preenchidos/respondidos pelos pais. A maioria dos instrumentos testaram a sua coerência interna $(n=9)$ e, em menor número, a reprodutibilidade teste-reteste $(n=$ 7) e concordância entre observadores $(n=2)$. A maioria dos questionários relatou a sua validade de conteúdo $(n=10)$ e validade de construção $(n=9)$, poucos analisaram a validade de critério $(n=2)$. Dos instrumentos analisados, seis questionários estão validados para a população portuguesa (cinco genéricos e um específico de doença oncológica).

Palavras-chave: Cuidados Paliativos; Pediatria; Qualidade de Vida; Questionários.

\section{ABSTRACT}

Introduction: Palliative care is closely linked to the concept of quality of life. In this work we will focus our interest on the need to assess quality of life in oncologic paediatric palliative care.

Objective: To describe/compare instruments for measuring quality of life in Oncologic paediatric palliative care.

Material and Methods: A literature review of the sever a instruments for measuring quality of life of children under palliative care, in English and Portuguese, between 2000 and 2013, was carried out in the recognized databases for this purpose.

Results: We found fifteen measuring instruments: 10 of them were generic and 5 specific. For each instrument the country of origin, the target age group, fill manner, number of evaluated dimensions, description of dimensions, and number of questions, psychometric properties and validation for the Portuguese language were identified.

Discussion: There has been a growing concern in measuring quality of life in pediatric age. Most measuring instruments were designed, in the United States, after 1994, coinciding with the World Health Organization definition of quality of life. As regards to age, most of the instruments were developed for children aged eight or more years old and there is no one to be answered only by the child. We can see that the majority of measuring instruments, namely the most current, seeking to involve the child in evaluating his/her own health related quality of life through auto-population $(n=10)$. However, there is still a substantial dependence on parents for the measurement

1. Faculdade de Medicina. Universidade de Coimbra. Coimbra. Portugal.

2. Unidade de Saúde Familiar de Terras de Souza. ACeS Tâmega II - Vale do Sousa Sul. Penafiel. Portugal.

3. Instituto de Patologia Geral. Faculdade de Medicina. Universidade de Coimbra. Coimbra. Portugal.

4. Faculdade de Economia. Universidade de Coimbra. Coimbra. Portugal.

$\triangle$ Autor correspondente: Marília Assunção Dourado. mdourado@fmed.uc.pt

Recebido: 01 de Abril de 2014 - Aceite: 29 de Junho de 2014 | Copyright @ Ordem dos Médicos 2015 
of health related quality of life of their children, despite studies demonstrates differences between the child and parents, on perception of health related quality of life. But, since many children are not able to provide data on health related quality of life either due to their age or because they are ill or with functional incapacity, the only possibility to get information about the health related quality of life of these children is to appeal to parents, who are asked to reflect on the lack of their child, or teenager. Thus full completion by parents of some measuring instruments may be justified. In Pediatrics measuring instruments of health related quality of life are multidimensional, often intended to measure the subjective point of view in relation to the impact of the disease and the treatment have on the physical, psychological and social well-being. Thus, the wide range of dimensions, the differences in number of dimensions and the number of questions between the various instruments tend to reflect the different stages of psychomotor development of the target population.

Conclusion: The most commonly measure dimensions were physical ability, emotional/psychological impact, social and at school impact, followed by pain and discomfort and activity level. The majority of instruments are designed for children aged over 8 years. A large number of the questionnaires are self-completion questionnaires. Others can be completed by parents. Most of the instruments tested their internal coherence $(n=9)$ and, in a small number, the test-retest reproducibility $(n=7)$ and agreement among observers $(n=2)$. Most of the questionnaires reported their content validity $(n=10)$ and construct validity $(n=9)$, few have examined the validity of criterion $(n=2)$. Considering the examined instruments, six questionnaires are validated for the Portuguese population (five generic and one specific for oncologic disease).

Keywords: Palliative Care; Pediatrics; Quality of Life; Questionnaires.

\section{INTRODUÇÃO}

Os cuidados paliativos (CP) visam melhorar a qualidade de vida (QV) dos doentes com doença ativa, progressiva, sem perspetiva de tratamento curativo, proporcionando alívio de sintomas físicos, suporte espiritual e psicossocial, devendo disponibilizar-se tudo aquilo que vá de encontro a essa finalidade. ${ }^{1,2}$ Para os CP pediátricos, citando o Royal College of Paediatrics and Child Health, ${ }^{3}$ há quatro grupos em que os CP podem ser aplicáveis (Tabela 1).

Os CP estão intimamente ligados à QV cujo conceito em saúde decorre dos novos paradigmas que têm influenciado as práticas e políticas de saúde nas últimas décadas ${ }^{4}$. A OMS define QV como a perceção individual sobre a sua posição na vida, num contexto cultural e num sistema de valores no qual o indivíduo vive, de acordo com os seus objetivos, expectativas e padrões, um conceito complexo multidimensional. ${ }^{5}$

Em saúde 'qualidade de vida' pode conceptualizar-se genericamente ou relacionando-a com a saúde (QVRS), definida pelo conhecimento subjetivo do impacto da doença e tratamento, suas representações através dos domínios funcional, de bem-estar físico, psicológico e social. ${ }^{6} \mathrm{~A}$ sua avaliação visa perceber como diferentes domínios são influenciados pelas características e tratamento da doença, constituindo-se como importante indicador para a avaliação do impacto da doença, da eficácia e eficiência dos tratamentos e intervenções; alocação de recursos à saúde; realização de estudos epidemiológicos, potenciando a produção e integração do conhecimento e o seu intercâmbio. Os instrumentos de medição da QVRS são úteis, quando aplicados individualmente, na identificação e priorização de problemas de saúde, no auxílio e partilha à tomada de decisão e na monitorização das alterações no estado de saúde dos doentes ou na resposta ao tratamento. ${ }^{7,8}$

Em pediatria, a medição da QVRS usa instrumentos genéricos e específicos. Os primeiros avaliam e comparam a QV de crianças saudáveis e doentes. Os segundos avaliam a QV do doente, recolhem informações sobre sintomas ou aspetos específicos de determinadas doenças e tendem a ser mais sensíveis para detectar alterações relacionadas com o tratamento. ${ }^{7} \mathrm{~A}$ utilização de instrumentos de medição em pediatria levanta algumas dificuldades relacionadas com a capacidade das crianças expressarem opiniões sobre a sua QVRS e de compreenderem o conceito de QVRS. Segundo alguns autores, as crianças são capazes de o fazer se questionadas apropriadamente, para a idade e nível cognitivo. ${ }^{4}$ Para resolver estes problemas construíram-se questionários e desenvolveram-se novas estratégias e métodos, com diferentes categorias de resposta, smile ou pictogramas.

A construção de instrumentos de medição para avaliar

Tabela 1 - Condições em que os Cuidados Paliativos Pediátricos podem ser aplicáveis e representar uma resposta para os doentes e suas famílias. Adaptado de Health guide to the development of children's palliative care services. Royal College of Paediatrics and Child (1997). ${ }^{3}$

Grupos

Condições em que o tratamento curativo pode ser possível, mas pode também ser falível. Os Cuidados Paliativos podem ser necessários durante períodos de prognóstico incerto e quando os tratamentos falham. Doentes em longos períodos de remissão ou em tratamentos curativos com sucesso não são incluídos.

Condições que requerem tratamento intensivo a longo prazo, com o objetivo de manter a participação em atividades normais da infância.

Condições progressivas para as quais os tratamentos são exclusivamente paliativos após o diagnóstico.

Condições que envolvem distúrbios severos e não progressivos que podem ser causa de suscetibilidade para outras complicações.
Patologia neoplásica

Falência cardíaca, hepática ou renal, irreversível

Fibrose Cística

Distrofia Muscular

Doença de Batten

Mucopolissacaridoses

Paralisia Cerebral Severa

Lesão Cerebral ou Medular 
a QV em pediatria tem limitações, como a definição das dimensões relevantes para descrever QVRS em crianças e adolescentes, que não são necessariamente as mesmas que os adultos valorizam. ${ }^{4} \mathrm{~A}$ criança raramente é chamada a expressar a sua opinião e, na maioria dos casos, a única forma de obter informação sobre a QV é recorrer a fontes complementares, como os pais, pedindo que reflitam sobre a perceção de QV da criança, o que não é necessariamente concordante. Por isso, os instrumentos que têm sido desenvolvidos, mais recentemente, têm valorizado mais o ponto de vista da criança, envolvendo-as mais diretamente nas decisões sobre si mesmas.

Assim, o objetivo deste trabalho é identificar os atuais instrumentos de medição de QV, em crianças até aos 18 anos, descrever o seu conteúdo e avaliar as suas propriedades psicométricas.

\section{MATERIAL E MÉTODOS}

Foi realizada uma pesquisa bibliográfica referente aos vários instrumentos de medição existentes para avaliar a perceção da QV das crianças em $\mathrm{CP}$, publicados em inglês e português, entre 2000 e 2013, com abstract disponível, utilizando as palavras-chave (termos MeSH) child, adolescent, paediatric, quality of life, instruments measuring, questionnaires, palliative care, cancer, oncology e advanced illness, nas bases de dados: PubMed, National Guideline Clearinghouse, Cochrane, PsycINFO, índex de revistas médicas portuguesas. Foram ainda consultados capítulos de livros de referência na área dos CP.
Consideraram-se critérios de inclusão e de exclusão, para os artigos a incluir no estudo.

Para cada instrumento identificou-se o país de origem, faixa etária a que se destina, modalidade de preenchimento, o número e a descrição das dimensões avaliadas, o número de perguntas, as propriedades psicométricas, e a validação para a população portuguesa.

\section{RESULTADOS}

Encontraram-se 147 artigos, mas apenas 41 foram incluídos no estudo (Fig. 1); e foram identificados 15 instrumentos de medição de QV em pediatria, dos quais 10 são instrumentos genéricos e cinco específicos para doenças oncológicas (Tabela 2), que seguidamente descrevemos.

\section{Child Health and IIIness Profile - Adolescent Edition (CHIP-AE) ${ }^{9}$}

Desenvolvido para descrever o estado físico e mental dos adolescentes. É constituído por cerca de 153 perguntas que medem dimensões como a satisfação com a saúde, desconforto (físico, emocional e limitações na atividade), doenças (agudas minor ou major, recorrentes, psicossociais, cirúrgica/médica prolongada), riscos (individuais ou relacionados com o tratamento), resiliência (envolvimento da família, resolução de problemas, atividade física, segurança em casa) e autorrealização (desempenho académico ou laboral, de acordo com os papéis sociais de cada grupo etário). Os sinais e sintomas da doença são reportados em relação à sua existência nas últimas quatro semanas.

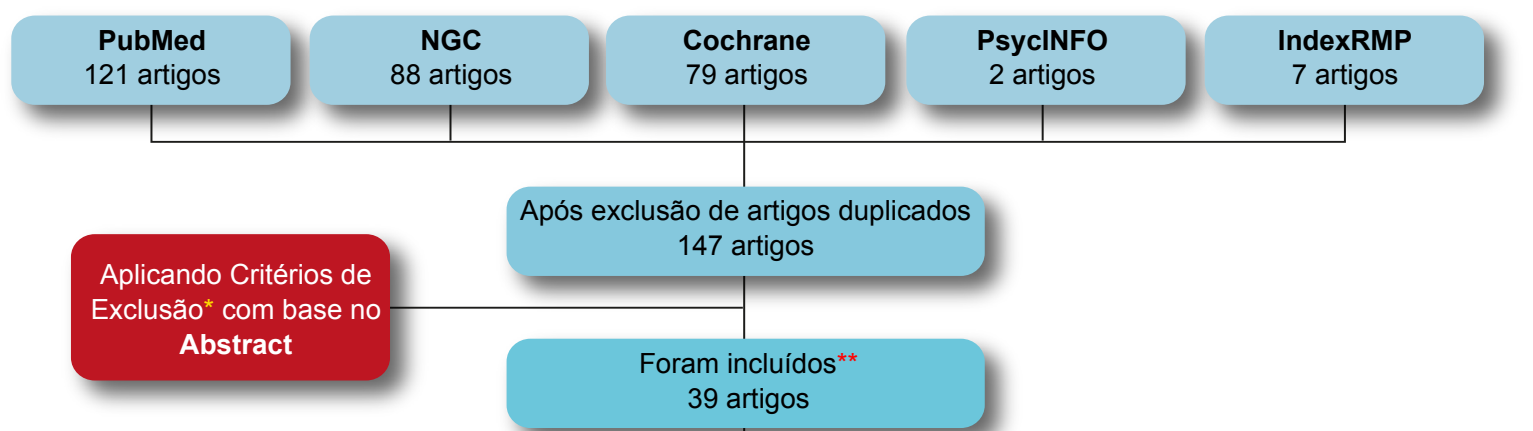

Foram incluídos mais 5 artigos considerados artigos chave

Número total de artigos*: 41 Número total de instrumentos de medição: 15

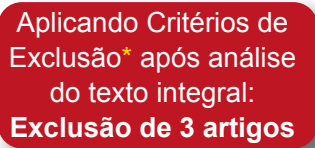

Aplicando Critérios de

ão* apos analise Exclusão de 3 artigos

Figura 1 - Resultados da pesquisa bibliográfica. A pesquisa bibliográfica foi realizada nas bases de dados: PubMed, National Guideline Clearinghouse, Cochrane, PsycINFO, entre outras. Após exclusão dos artigos encontrados em duplicado, foram selecionados 147 artigos dos quais, após a aplicação dos critérios de exclusão, 41 foram incluídos no estudo. Encontraram-se 15 instrumentos de medição de qualidade de vida em idade pediátrica, dos quais 10 são instrumentos genéricos e 5 são instrumentos específicos de qualidade de vida em doenças oncológicas.

*Artigos incidindo em instrumentos de medição de QV originalmente concebidos para utilização em adultos ou na população em geral; Artigos que referem o uso de escalas funcionais e listas de verificação de sintomas; Artigos que utilizam instrumentos de medição específicos para asma, epilepsia, hemofilia, fibrose quística, diabetes, entre outras doenças; Artigos de revisão, ensaios clínicos e relatos de caso.

** Artigos que incluem instrumentos de medição da QV, especificamente para crianças e adolescentes até aos 18 anos de idade; incluem instrumentos que podem ser preenchidos pelo próprio, por fontes complementares (pais, cuidadores ou profissionais de saúde) ou ambos; descrevem o desenvolvimento ou a validação de um instrumento de medição; incluem instrumento de medição avalie (pelo menos um domínio) QV na população pediátrica; se referem às propriedades psicométricas dos instrumentos de medição. 
Tabela 2 - Instrumentos genéricos e específicos de medição da qualidade de vida em idade pediátrica. São apresentadas, resumidamente, as características dos diferentes instrumentos de medição designadamente quanto à modalidade de preenchimento, número e descrição das dimensões avaliadas e validação para a população Portuguesa.

Instrumentos genéricos de medição da qualidade de vida em idade pediátrica

\begin{tabular}{|c|c|c|c|c|c|}
\hline Instrumento de Medição & País de Origem & Faixa Etária (anos) & $\begin{array}{l}\text { Modalidade de } \\
\text { Preenchimento }\end{array}$ & $\begin{array}{l}\text { Número de } \\
\text { Dimensões }\end{array}$ & $\begin{array}{l}\text { Número de } \\
\text { Perguntas }\end{array}$ \\
\hline $\begin{array}{l}\text { CHIP-AE - (Child Health and IIIness } \\
\text { Profile) })^{9}\end{array}$ & EUA & $11-17$ & Autopreenchimento & 6 & 153 \\
\hline CHQ - (Child Health Questionaire) $)^{11}$ & EUA & $10-18$ & Autopreenchimento & 11 & 87 \\
\hline & & $5-18$ & Pais & & 50 ou 28 \\
\hline $\begin{array}{l}\text { COOP - (Dartmouth COOP Functional } \\
\text { Health Assessment Charts) })^{13,14}\end{array}$ & EUA & $12-21$ & Autopreenchimento & 6 & 6 \\
\hline FS II (R) - Functional Status II (R) ${ }^{17}$ & EUA & $0-16$ & $\begin{array}{l}\text { Pais } \\
\text { (Entrevista) }\end{array}$ & 3 & $\begin{array}{c}14 \\
\text { (versão curta) } \\
43 \\
\text { (versão longa) }\end{array}$ \\
\hline KIDSCREEN-52 ${ }^{19}$ & $\begin{array}{l}\text { Internacional } \\
\text { (Áustria, França, Alemanha, } \\
\text { Grécia, Holanda, Hungria, Irelanda, } \\
\text { Polónia, Espanha, Suécia, Suiça, } \\
\text { República Checa e Reino Unido) }\end{array}$ & $8-18$ & $\begin{array}{l}\text { Autopreenchimento } \\
\text { Pais }\end{array}$ & 10 & 52 \\
\hline $\begin{array}{l}\text { KINDL - (Fragebogen zur Lebensqualitat } \\
\text { von Kindern \& Jugendlichen })^{20}\end{array}$ & Alemanha & $8-16$ & $\begin{array}{l}\text { Autopreenchimento } \\
\text { Pais }\end{array}$ & 6 & 24 \\
\hline $\begin{array}{l}\text { PedsQL } 4.0 \text { - (Pediatric Quality of Life } \\
\text { Inventory Version } 4.0)^{22,23}\end{array}$ & EUA & $\begin{array}{l}5-18 \\
2-18\end{array}$ & $\begin{array}{l}\text { Autopreenchimento } \\
\text { Pais }\end{array}$ & 4 & 23 \\
\hline $\begin{array}{l}\text { WCHMP - (Warwick Child Health and } \\
\text { Morbidity Profile })^{25}\end{array}$ & Reino Unido & $0-5$ & Pais & 10 & 16 \\
\hline $\begin{array}{l}\text { 16D - (16-Dimensional Health-related } \\
\text { Quality of Life Measure })^{26}\end{array}$ & Finlândia & $12-15$ & Autopreenchimento & 16 & 16 \\
\hline $\begin{array}{l}\text { 17D - }(17-\text { Dimensional Health-related } \\
\text { Measure })^{27}\end{array}$ & Finlândia & $8-11$ & Autopreenchimento & 17 & 17 \\
\hline
\end{tabular}

Instrumentos específicos de medição da qualidade de vida em crianças com doença oncológica

\begin{tabular}{|c|c|c|c|c|c|}
\hline Instrumento de Medição & País de Origem & Faixa Etária (anos) & $\begin{array}{l}\text { Modalidade de } \\
\text { Preenchimento }\end{array}$ & $\begin{array}{l}\text { Número de } \\
\text { Dimensões }\end{array}$ & $\begin{array}{l}\text { Número de } \\
\text { Perguntas }\end{array}$ \\
\hline $\begin{array}{l}\text { BASES - Behavioral Affective and Somatic } \\
\text { Experiences Scale })^{28}\end{array}$ & EUA & $5-17$ & $\begin{array}{l}\text { Autopreenchimento } \\
\text { Pais } \\
\text { Eq. Enfermagem }\end{array}$ & 5 & $\begin{array}{l}14 \\
38 \\
38\end{array}$ \\
\hline $\begin{array}{l}\text { MPQOL - (The Miami Pediatric Quality of } \\
\text { Life Questionnaire) }{ }^{29}\end{array}$ & EUA & $1-18$ & Pais & 3 & 56 \\
\hline $\begin{array}{l}\text { PCQL-32 - (The Pediatric Cancer Quality } \\
\text { of Life Inventory })^{30}\end{array}$ & EUA & $\begin{array}{c}\text { 8-12 (crianças) } \\
\text { 13-18 (adolescentes) }\end{array}$ & $\begin{array}{l}\text { Autopreenchimento } \\
\text { Pais }\end{array}$ & 5 & 32 \\
\hline $\begin{array}{l}\text { POQOLS - (The Pediatric Oncology } \\
\text { Quality of Life Scale) })^{31}\end{array}$ & EUA & $3-18$ & Pais & 3 & 21 \\
\hline $\begin{array}{l}\text { PPSC - (The Play Performance Scale for } \\
\text { Children })^{33,34}\end{array}$ & EUA & $0,5-16$ & Pais & 1 & 1 \\
\hline
\end{tabular}


Instrumentos genéricos de medição da qualidade de vida em idade pediátrica

Descrição das Dimensões

Desconforto, doenças, satisfação com a saúde, autorealização, riscos e resiliência

Capacidade física, a perceção global de saúde, dor, impacto social, impacto emocional e comportamento, saúde mental, comportamento geral, autoestima, impacto emocional dos pais,

impacto temporal nos pais, impacto familiar

Bem-estar físico, saúde mental, escola, suporte social, comunicação familiar, hábitos de saúde

Capacidade física, psicológica e social

$0-2$ anos: capacidade de resposta

2-3 anos: atividade

4 anos: funcionamento interpessoal

Saúde e atividade física, sentimentos, estado de humor geral, auto perceção, tempo livre, família e ambiente familiar, questões económicas, amigos, ambiente escolar e aprendizagem, provocação

Bem-estar físico, bem-estar psicológico, autoestima, família, amigos e escola

Desempenho físico,emocional, social e escolar

Saúde em geral, doença aguda ligeira, comportamento, acidentes, doença aguda grave, admissão hospitalar, imunização, doenças crónicas, status funcional e qualidade de vida relacionada com a saúde

Mobilidade, visão, respiração, sono, alimentação, evacuação, discurso, escola e passatempos, amigos, aparência física, função mental, desconforto, sintomas, depressão, ansiedade e vitalidade

Mobilidade, visão, respiração, sono, alimentação, evacuação, discurso, escola e passatempos, amigos, aparência física, desconforto, sintomas, depressão, ansiedade, vitalidade, capacidade de compreensão, capacidade de aprendizagem e memória
Teste-Reteste

Coerência Interna
Validade

Coerência Interna Teste-Reteste

Coerência Interna

Construção

$\operatorname{Sim}(16)$

Validação População Portuguesa

$\operatorname{Sim}(10)$

Construção

Não

Não

Teste-Reteste

Construção

$\operatorname{Sim}(4)$

Critério

$\begin{array}{llr}\begin{array}{l}\text { Coerência Interna } \\ \text { Teste-Reteste }\end{array} & \begin{array}{l}\text { Conteúdo } \\ \text { Construção }\end{array} & \operatorname{Sim}(21) \\ \text { Coerência Interna } & \begin{array}{l}\text { Conteúdo } \\ \text { Construção }\end{array} & \operatorname{Sim}(24) \\ \text { Teste-Reteste } & \text { Construção } & \text { Não }\end{array}$

Teste-Reteste

Conteúdo

Não

Teste-Reteste

Conteúdo

Não

Instrumentos específicos de medição da qualidade de vida em crianças com doença oncológica

Descrição das Dimensões

Desconforto somático, compliance, humor e comportamento, interações, atividade

Auto-competência, estabilidade emocional e competência social

Desempenho físico, sintomas relacionados com a doença e com o tratamento, desempenho psicológico, desempenho social e desempenho cognitivo.

Desempenho físico e limitação na atividade, sofrimento emocional, resposta ao tratamento médico atual

Nível de atividade
Fiabilidade

Validade

Validação População Portuguesa 
A resposta é dada de acordo com o nível de concordância numa escala tipo Likert. O tempo médio de preenchimento é de 30 minutos. Está validado para português. ${ }^{10}$

\section{Child Health Questionnaire (CHQ) ${ }^{11}$}

Questionário que avalia as componentes física, psicológica e social. Foi desenvolvido para crianças (idade igual ou superior a cinco anos de idade) e adolescentes. Existem questionários específicos para preenchimento parental (CHQ-PF50 e PF28-versão curta) e de autopreenchimento por crianças com mais de 10 anos de idade (CHQ-CF87). Incluí as dimensões: capacidade física, perceção global da saúde, saúde mental, comportamento global, impacto da incapacidade física a nível social, emocional e comportamental, dor ou desconforto, impacto da doença nos pais, impacto e limitações nas atividades familiares. As pontuações são convertidas para escala de 0 a 100. Uma pontuação mais elevada indica um melhor estado de saúde. $O$ tempo de preenchimento não foi reportado. O CHQ-PF50 e PF28 estão traduzidos para português mas não validados. ${ }^{12} \mathrm{O}$ CHQ-CF 87 não está traduzido nem validado para português.

\section{Dartmouth COOP Functional Health Assessment Charts $(\mathrm{COOP})^{13-15}$}

Consiste em diagramas simples, de preenchimento e interpretação fáceis. Existem questionários vocacionados para adolescentes cujas dimensões abordadas são: aptidão física, saúde mental, função social (escola, família e apoio social), hábitos de saúde (prejudiciais e benéficos) e autoimagem, saúde em geral e dor. Há versões mais simplificadas para crianças, com nove perguntas, que avaliam três dimensões: função (preparação física, energia, emoções, acerca de ti, 'como te sentes'), saúde em geral, escola e casa (coisas que faço todos os dias, relação com os outros e família). Estes últimos questionários têm fiabilidade e validade menores. As respostas são dadas de acordo com o nível de concordância usando uma escala do tipo Likert, com ilustrações, nivelada 1 a 5 . O tempo de preenchimento é de cerca de 1 - 2 minutos para o COOPcrianças, e de 4 - 5 minutos para o COOP-adolescentes. Já foi testado e validado em Portugal. ${ }^{16}$

\section{Functional Status II (R) (FS II (R) ${ }^{17}$}

Descreve o status funcional da criança nas duas semanas anteriores, segundo os aspetos físicos, psicológicos e sociais. Existe uma versão longa constituída por 43 perguntas e outra curta de 14 questões, ambas de preenchimento por entrevista aos pais. O preenchimento da versão longa demora em média 15 - 30 minutos, a curta demora menos de 10 minutos $^{18}$.

\section{KIDSCREEN-52 4,19}

Instrumento genérico, pode ser utilizado para medição, avaliação e monitorização. É aplicável em crianças e adolescentes dos 8 aos 18 anos de idade e aos pais, no âmbito da saúde e da doença crónica. É um questionário de autopreenchimento, que demora de 10 a 15 minutos. Foi desenvolvido no âmbito do projeto Europeu "Screening and Promotion for Health-Related Quality of Life in Children and Adolescents - A Europen Public Health Perspective", da Comissão Europeia, para medir dez dimensões: saúde e atividade física, sentimentos, estado de humor geral, auto perceção, tempo livre, família e ambiente familiar, questões económicas, amigos, ambiente escolar e aprendizagem e provocação (bullying). Foi testado e validado para português. ${ }^{4}$

\section{Fragebogen zur Lebensqualitat von Kindern \& Jugendlichen (KINDL) ${ }^{20}$}

Desenvolvido para medir a QV em pediatria, sob qualquer condição de saúde, podendo ser preenchido pela criança ou pelos pais. São realizadas 24 perguntas, pontuadas de 1 a 5, procurando medir seis dimensões: bem-estar físico, bem-estar emocional, autoestima, família, amigos e escola. As pontuações, total e por dimensão são apresentadas numa escala de orientação positiva de 0 (pior) a 100 (melhor). O tempo médio de preenchimento é de 5 a 6 minutos. Está validado para português sendo designado por Questionário Kid-KINDL (crianças ou pais), destinado crianças dos 8 e os 12 anos, e Questionário Kiddo-KINDL (adolescentes ou pais), para as crianças entre 13 - 16 anos de idade. ${ }^{21}$

\section{Pediatric Quality of Life Inventory, version 4.0 (PedsQL 4.0) 22,23}

Dirigido a crianças e adolescentes, de idades compreendidas entre os 2 e os 18 anos de idade. Consiste num conjunto de escalas, aplicáveis a populações saudáveis, assim como a populações com patologia aguda ou crónica. As dimensões avaliadas são: funcionamento físico (oito itens), funcionamento emocional (cinco itens), funcionamento social (cinco itens) e o funcionamento na escola (cinco itens). É constituído por um total de 23 perguntas, demorando cerca de 4 - 5 minutos a preencher. A análise dos resultados pode realizar-se através de um resultado total e de dois sub-resultados: um específico da saúde física e outro relativo à saúde psicossocial. As instruções aconseIham que o respondente pense em cada um dos problemas apontados em termos da sua ocorrência durante o mês anterior e as respostas estão organizadas numa escala tipo Likert com cinco opções. As pontuações por dimensão são apresentadas numa escala de orientação positiva de 0 (pior QV) a 100 (melhor QV). O PedsQL possui várias versões adequadas a diferentes períodos do desenvolvimento, assim como formatos de autopreenchimento (para crianças e adolescentes dos 5 aos 18 anos) e preenchimento pelos pais (para crianças e adolescentes entre os 2 e os 18 anos). Está validado para português sob a designação Inventário Sobre a Qualidade de Vida Pediátrica, versão 4.0, inquérito às crianças e inquérito aos pais sobre os seus filhos (5 - 7 anos) e Inventário Sobre a Qualidade de Vida Pediátrica, versão 4.0 , inquérito às crianças e inquérito aos pais sobre os seus filhos (8 - 12 anos). ${ }^{24}$ 
Warwick Child Health and Morbidity Profile (WCHMP) ${ }^{25}$

O WCHMP mede a perceção dos pais sobre o estado de saúde e de doença dos seus filhos, sendo aplicado em crianças entre os 0 e os 5 anos de idade. É um instrumento simples, levando apenas 10 minutos para ser concluído. É composto por 10 domínios: saúde em geral, doença aguda ligeira, comportamento, acidente, doença aguda grave, admissão hospitalar, imunização, doenças crónicas, status funcional e qualidade de vida relacionada com a saúde. Este instrumento ainda não está validado para português.

\section{6-Dimensional Health-related Quality of Life Measure} $(16 D)^{26}$

Consiste num instrumento de medição genérico de QVRS para adolescentes dos 12 aos 15 anos, construído a partir do instrumento 15D para adultos. É de autopreenchimento e foi testado em quatro populações de crianças: saudáveis em idade escolar; que aguardam transplante de órgãos; com displasias músculo-esqueléticas e com epilepsia. O instrumento é composto por 16 questões de escolha múltipla, cada uma representando uma dimensão relacionada com a saúde. Os estudos iniciais mostram fiabilidade e validade aceitáveis. O 16D tem provado ser capaz de diferenciar a QVRS de adolescentes saudáveis, bem como de doentes com vários diagnósticos.

\section{7-Dimensional Health-related Measure (17D) ${ }^{27}$}

O 17D foi concebido para medir a QV em pré-adolescentes, tendo por base o 16D (para adolescentes) e a 15D (para adultos). As dimensões utilizadas baseiam-se no 16D, mas ajustado para uma faixa etária mais jovem. Foi testado em três grupos de crianças, com idades entre 8 a 11 anos, crianças: saudáveis; sobreviventes de transplante de órgãos e crianças com displasias músculo-esqueléticas (Tabela 3).

Além dos instrumentos genéricos de avaliação da $Q V$, existem outros instrumentos especificamente desenhados para medir a QV em crianças com cancro, tendo sido identificados cinco que passamos a descrever.

\section{Behavioral Affective and Somatic Experiences Scale (BASES) $^{28}$}

Desenvolvido para avaliar as componentes física, afetiva e comportamental das crianças entre os 5 e os 17 anos na fase aguda pós transplante de medula óssea. Trata-se de um instrumento constituído por 38 perguntas que avaliam cinco dimensões: desconforto somático, compliance, humor/comportamento, interação e atividade. Pode ser preenchido pela própria criança (existindo uma versão curta de apenas 14 questões) ou pelos pais ou equipa de enfermagem. Não está validado para a população pediátrica portuguesa.

\section{The Miami Pediatric Quality of Life Questionnaire (MPQOL) $^{29}$}

É um instrumento orientado para medir a QV de crianças com cancro, de preenchimento pelos pais. É constituí- do por 56 questões que procuram medir três dimensões principais: auto competência, estabilidade emocional e competência social. Este questionário demonstrou ter capacidade de discriminar a QV de crianças com diferentes tipos de cancro, mostrando uma maior inclusão sobre preocupações psicológicas e sociais como fatores principais na determinação da QVRS em crianças.

\section{The Pediatric Cancer Quality of Life Inventory (PCQL- 32) ${ }^{30}$}

É um questionário específico para avaliar a QV de doentes oncológicos em idade pediátrica, com versões para crianças e para adolescentes. É constituído por 32 perguntas, abrangendo cinco domínios: desempenho físi$\mathrm{co}$, sintomas relacionados com a doença e tratamento, desempenho psicológico, desempenho social e desempenho cognitivo. As respostas estão organizadas numa escala tipo Likert com quatro opções. Desconhece-se validação para a população portuguesa.

\section{The Pediatric Oncology Quality of Life Scale (POQOLS) ${ }^{31}$}

Inclui 21 itens distribuídos por três dimensões: funcionamento físico e restrição das atividades normais; problemas emocionais e resposta ao tratamento médico atual. A resposta é dada numa escala ordinal de sete pontos entre 'nunca' e 'muito frequentemente'. A resposta é dada pelos pais das crianças com cancro ou que a ele sobreviveram, com idade compreendida entre os 3 e os 18 anos. Resultados elevados indicam uma baixa QVRS. Encontrase validada para a população portuguesa. ${ }^{32}$

\section{The Play Performance Scale for Children (PPSC) (3,34 $^{3,}$}

É uma escala de uma só pergunta desenhada para avaliar o nível de atividade de crianças com cancro. Classificase de 1 a 100, baseando-se no nível de atividade e desempenho em jogos. Esta escala apresenta a vantagem de ser muito curta e, por isso, facilmente aplicável, mesmo em crianças muito debilitadas. Não se encontra validada para a população portuguesa.

Para podermos estimar, rapidamente, quais as dimensões avaliadas por cada instrumento ou quantos/quais instrumentos existem para medir determinada dimensão ou ainda qual ou quais as dimensões mais medidas, elaborámos a Tabela 3 onde são identificadas todas as dimensões avaliadas e o(s) instrumento(s), genérico ou específico que as avaliam, com base nas descrições de cada instrumento de medição (Tabela 3).

\section{DISCUSSÃO}

Os resultados desta revisão sistemática mostram que tem havido uma crescente preocupação em medir a QV em idade pediátrica. A maioria dos instrumentos de medição foi desenhada após 1994, coincidindo temporalmente com a definição de QV da OMS. Desde então, a produção de instrumentos de medição de QVRS para pediatria tem aumentado. Inicialmente desenvolveram-se sobretudo instrumentos genéricos de medição de QVRS e só mais tarde 
Tabela 3 - Instrumentos de medição e dimensões avaliadas. Dimensões avaliadas pelos instrumentos genéricos e específicos de qualidade de vida para crianças com patologia oncológica.

\begin{tabular}{l|l}
\hline Instrumentos de Medição Genéricos &
\end{tabular}

\section{Dimensões}

\section{Instrumentos de Medição Genéricos}

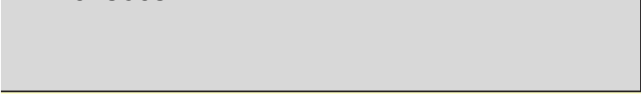

CHIP-AE

CHQ

Capacidade Física

Dor, sintomas ou desconforto

Atividades diárias (incluindo nível de atividade)*

Satisfação com a saúde e vitalidade

Perceção global de saúde

Doença**

Hábitos de saúde ${ }^{* * *}$

Autorealização

Autoestima, aparência física

Riscos e acidentes

Resiliência

Impacto social

Impacto psicológico/ emocional

Saúde Mental

Depressão e Ansiedade

Humor

Capacidade cognitiva****

Comportamento

Bullying

Preocupação dos pais com a doença

Família*****

Escola

Amigos

Tempos Livres

Questões económicas

Admissão hospitalar

Resposta ao tratamento

*Atividades diárias incluí mobilidade, visão, respiração, sono, alimentação, evacuação, discurso; ** Doença aguda, crónica, ligeira a severa; *** Hábitos de saúde (incluindo vacinação; ${ }^{* * * *}$ Capacidade cognitiva, incluindo aprendizagem, concentração e memória; ***** Família, incluindo coesão familiar, comunicação na família, impacto familiar. 


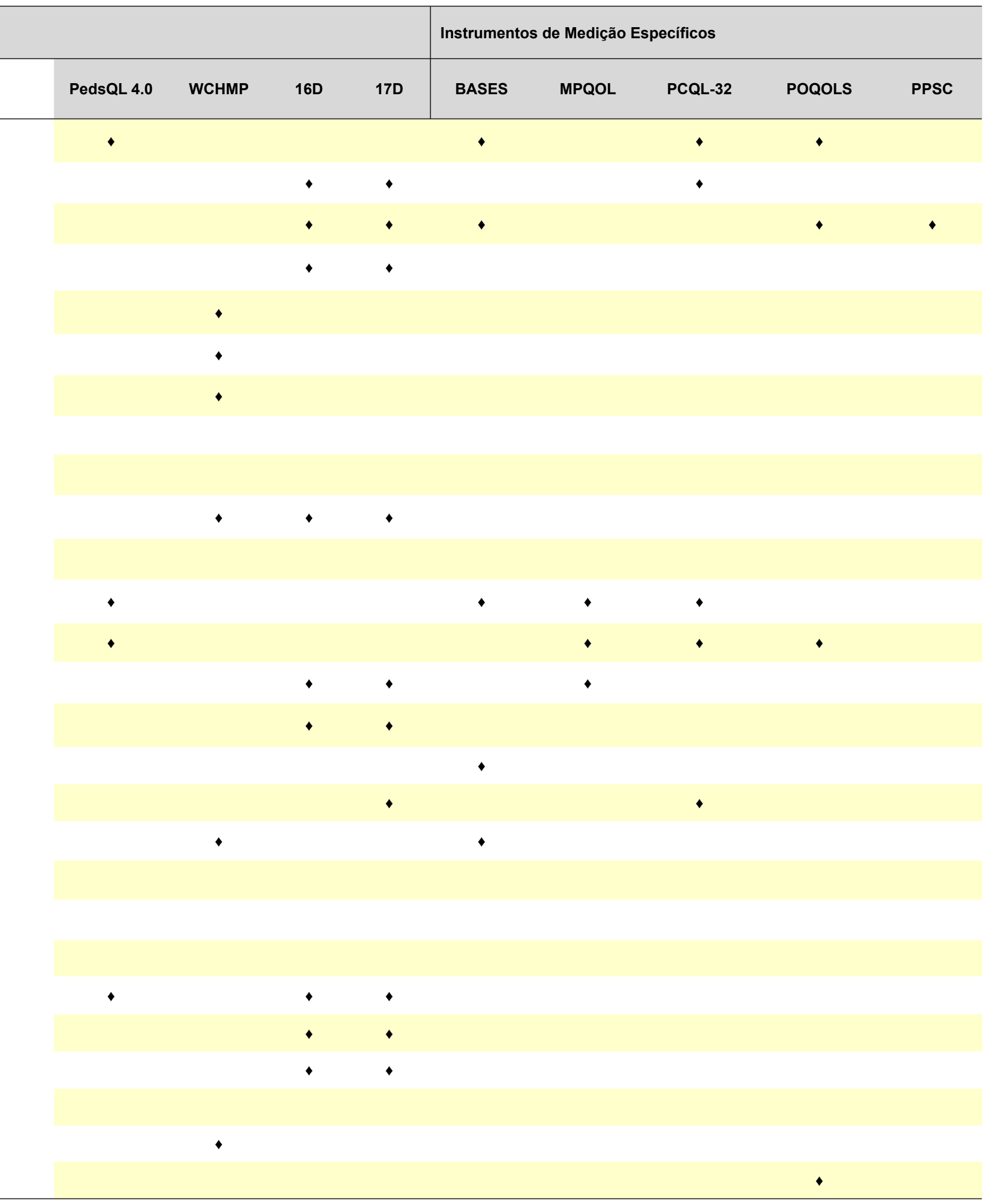


começaram a surgir instrumentos de medição específicos, nomeadamente para crianças com cancro.

A maioria dos instrumentos foi desenvolvida nos Estados Unidos $(n=10)$, e todos os instrumentos específicos de doença oncológica são provenientes daquele país. Apenas um foi desenvolvido simultaneamente em mais de um país (KIDSCREEN-52). Deste modo, a língua original da grande maioria destes instrumentos é o inglês.

Relativamente à faixa etária, a maioria dos instrumentos foi desenvolvida para crianças com idade igual ou superior a oito anos. Apenas três instrumentos genéricos e dois específicos foram direcionados para a primeira infância.

Quanto ao preenchimento, quatro instrumentos genéricos são de exclusivo autopreenchimento pela criança, dois instrumentos genéricos e três específicos são de preenchimento exclusivo pelos pais e quatro instrumentos genéricos e dois específicos pelos pais e/ou pela criança, existindo um instrumento específico que pode ser preenchido pela equipa de enfermagem. Não existe nenhum instrumento específico para preenchimento apenas pela criança.

Assim, podemos verificar que a maioria dos instrumentos de medição, sobretudo os mais atuais, procuram envolver a criança na avaliação da sua própria QVRS através do autopreenchimento $(n=10)$. Porém, há ainda uma dependência substancial dos pais para a medição da QVRS dos seus filhos, apesar dos estudos que demonstram diferenças na perceção de QVRS entre a criança e os pais. ${ }^{35}$ Algumas crianças não têm capacidade de fornecer dados sobre a sua QVRS, quer devido à idade quer porque estão doentes ou com incapacidade funcional. Assim, a única possibilidade de obter informação sobre a QVRS destas crianças é o recurso aos pais, a quem é pedido que reflitam sobre a perceção da criança, ou adolescente, podendo, assim, justificar-se o preenchimento integral pelos pais de alguns instrumentos de medição.

O número de dimensões medidas pelos instrumentos genéricos variou entre 3 (FSII (R)) e 17 (17D) e nos instrumentos específicos entre 1 (PPSC) e 5 (BASES e PCQL32). A especificidade e exigência da medição da $Q V$ em crianças/adolescentes com cancro, pode justificar esta diferença no número de dimensões.

Quanto ao número de perguntas nos instrumentos genéricos varia entre 6 (COOP) e 153 (CHIP-AE), e nos instrumentos específicos variou entre 1 (PPSC) e 56 (MPQOL).

As dimensões mais comummente medidas são: Capacidade física; Impacto emocional/psicológico; Impacto social; Escola (Tabela 3).

As dimensões 'dor, sintomas e desconforto' assim como 'atividades diárias incluindo o nível de atividade' são medidas por cinco instrumentos distintos (genéricos e específicos).

Focando-nos apenas nos instrumentos de medição da QV de crianças com cancro, verificamos que as dimensões mais medidas são: capacidade física $(n=3)$, atividades diárias incluindo nível de atividade $(n=3)$, impacto social $(n=$ $3)$ e impacto emocional/psicológico $(n=3)$. Apenas um instrumento mede a resposta ao tratamento médico instituído no momento (POQOLS). Dada a preocupação com os sintomas físicos, impacto social e emocional, é provável que os instrumentos específicos de doença oncológica possam ser mais úteis para medir $\mathrm{QV}$ nas crianças em $\mathrm{CP}$, do que os instrumentos genéricos. Mas, não existe ainda nenhum instrumento de medição especificamente concebido para medir QV em crianças com cancro em $C P$.

Em relação aos instrumentos de medição que estão validados para português, verifica-se que são maioritariamente instrumentos genéricos (CHIP-AE, COOP, KIDSCREEN-52, KINDL e PedsQL 4.0). Na verdade, existe apenas um instrumento de medição específico de QV em crianças com cancro validado para a população portuguesa - o POQOLS.

Foram avaliados critérios psicométricos de qualidade, nomeadamente, a fiabilidade e a validade, de cada instrumento de medição. Quanto à fiabilidade, a maioria dos instrumentos testaram a sua coerência interna $(n=9)$ e, em menor número, a reprodutibilidade teste-reteste $(n=7)$ e concordância entre observadores $(n=2)$.

Em relação à validade, a maioria dos questionários relatou a sua validade de conteúdo $(n=10)$ e validade de construção $(n=9)$, mas poucos analisaram a validade de critério $(n=2)$. A escassa referência quanto à responsividade dos diferentes instrumentos remete para alguma limitação quanto à capacidade de monitorização da $\mathrm{QV}$ das crianças ao longo do tempo, usando aqueles instrumentos.

Os resultados do presente estudo sugerem que os instrumentos de medição de QVRS em pediatria são geralmente instrumentos multidimensionais, destinados a medir o ponto de vista subjetivo em relação ao impacto que a doença e o tratamento têm sobre o bem-estar físico, psicológico e social. Assim, a grande variedade de dimensões, as diferenças no número de dimensões e no número de perguntas entre os diversos instrumentos tendem a refletir os diferentes estádios de desenvolvimento psicomotor da população-alvo de cada instrumento. Deste modo, atendendo ao estádio de desenvolvimento dever-se-á considerar diversos aspetos, nomeadamente, a capacidade de compreensão verbal/escrita, compreensão e gestão do tempo, dimensões apropriadas à faixa etária, e perguntas sobre QV que sejam relevantes para a idade das crianças, para as quais o questionário é desenhado.

As principais limitações, identificadas, dos vários instrumentos de medição são:

- Confusão quanto à definição de QV;

- Heterogeneidade no número e conteúdo de dimensões;

- Disponibilidade limitada de instrumentos de medição específicos para doenças oncológicas;

- Número escasso de instrumentos validados para a população pediátrica portuguesa, nomeadamente para doença oncológica;

- Discrepâncias entre a avaliação pela criança e pelos pais;

- Disponibilidade limitada de medidas de autopreenchimento pela criança. 


\section{CONCLUSÃO}

Existem vários instrumentos de medição de $Q V$ em idade pediátrica, sendo a maioria instrumentos genéricos, que poderão ser usados para avaliar a QVRS em crianças com cancro, embora já existam alguns instrumentos específicos para avaliar a QV neste sub-grupo muito específico de crianças. Dada a preocupação com os sintomas físicos, impacto social e emocional, é provável que os instrumentos de medição específicos para cancro possam ser mais úteis para medir a qualidade QVRS nas crianças em CP do que os instrumentos genéricos. No entanto, não existe ainda nenhum instrumento de medição especificamente concebido para medir QVRS em crianças com cancro sob CP.

Os avanços nos cuidados de saúde, juntamente com a rápida evolução no domínio dos resultados de medições em saúde orientadas para o doente implicam a necessidade de aperfeiçoar os instrumentos de medição da QVRS

\section{REFERÊNCIAS}

1. World Health Organization. Cancer pain relief and palliative care. Geneve: WHO; 1990.

2. Associação Portuguesa de Cuidados Paliativos APCP. Organização de Serviços em Cuidados Paliativos - Recomendações da ANCP. Lisboa: APCP; 2006.

3. Association for Children with Life-threating or Terminal conditions and theirs Families, Royal College of Paediatrics and Child Health. A guide to the development of children's palliative care services. Edinburgh: Association for Children with Life-threating or Terminal conditions and theirs: 1997.

4. Gaspar T, Matos MG. Qualidade de vida em crianças e adolescentes: versão portuguesa dos instrumentos KIDSCREEN 52. Cruz Quebrada: Aventura Social e Saúde; 2008.

5. World Health Organization. The development of World Health Organization Quality of Life Assessement Instrument (the WHOQoL). Heidleberg: Springer-Verlag; 1994.

6. Ribeiro J. Qualidade de vida e doença oncológica. In: Dias M, Durá E. Territórios da Psicologia Oncológica. Lisboa: Climepsi Editora; 2002; p. 75-98.

7. Fayers PM, Machin D. Quality of life: assessment, analysis and interpretations. West Sussex: John Wiley \& Sons Ltd; 2000.

8. Higginson IJ, Carr AJ. Measuring quality of life: using quality of life measures in the clinical setting. BMJ. 2001;322:1297-300.

9. Starfield B, Bergner M, Ensminger M, Riley AW, Green BF, Ryan S. Child Health and IIIness Profile-Adolescent Edition (CHIP-AE). Baltimore: The Johns Hopkins University; 2000.

10. Rodrigues MA, Apóstolo JLA. Portuguese adaptation of the Child Health and IIIness Profile, Child Edition (CHIP-AE). Rev Enferm Ref. 2010;2:121-6.

11. Landgraf JM, Abetz LN. The CHQ User's Manual. Boston: The Health Institute, New England Medical Center; 1996.

12. Healhtactchq.com [homepage na Internet]. Boston, MA: HealthAct, Inc.; c2014; [Consultado 2013 Fev 20]. Disponível em: http://www. healthactchq.com/pdf/chq-t.php.

13. Wasson JH, Kairys SW, Nelson EC, Kalishman N, Baribeau P. A short survey for assessing health and social problem of adolescents. Dartmaounth Primary Care Cooperative Information Project (The COOP). J Fam Pract. 1994;38:489-94.

14. Wasson JH, Kairys SW, Nelson EC, Kalishman N, Baribeau P, Wasson E. Adolescent health and social problems. A method for detection and early management. The Dartmouth Primary Care Cooperative Information Project (COOP). Arch Fam Med. 1995;4:51-6.

15. qol.thoracic.org [homepage na Internet] American Thoracic Society - Quality of Life Resource, Inc; c2007; [Consultado 2013 fev 20]. Disponível em: http://qol.thoracic.org/sections/instruments/ae/pages/ coop.html.

16. Ferreira PL. A medição do estado de saúde de crianças. Saúde Infant. 1992;14:157-63.

17. Stein RE, Jessop DJ. Functional Status II (R). A measure oh child health status. Med Care. 1990;28:1041-55. em crianças com cancro, assim como a eminente necessidade de validação desses instrumentos para a população portuguesa.

\section{CONFIDENCIALIDADE DOS DADOS}

Os autores declaram ter seguido os protocolos do seu centro de trabalho acerca da publicação dos dados de doentes.

\section{CONFLITOS DE INTERESSE}

Os autores declaram não ter qualquer conflito de interesse relativamente ao presente artigo.

\section{FONTES DE FINANCIAMENTO}

Este trabalho não recebeu qualquer contribuição e subsídio ou bolsa.

18. qol.thoracic.org [homepage na Internet] America Thoracic Society - Quality of Life Resource, Inc; c2007; [Consultado 2013 Fev 20] Disponível em: http://qol.thoracic.org/sections/instruments/fj/pages/ functionalstatusii-r.html

19. Ravens-Sieberer U, Gosh A, Rajmil L, Erhart M, Bruil J, Power M, et al. The Kidscreen-52 quality of life measure for children and adolescents: psychometric results from a crosscultural survey in 13 European countries. Value Health. 2008;11:645-58.

20. Ravens-Sieberer U, Bullinger M. Assessing the health related quality of life in chronically ill children with the German KINDL: first psychometric and content-analytical results. Qual Life Res. 1998;7:399-407.

21. Ferreira $P$, Almeida $M$, Pisco $M$, Cavalheiro $L$. Qualidade de vida de crianças e adolescentes. Adaptação cultural e validação da versão portuguesa do KINDL®. Acta Pediatr Port. 2006;37:125-44.

22. Varni JW, Seid M, Rode CA. The PedsQL: Measurement model for pediatric quality of life inventory. Med Care. 1999;37:126-39.

23. Varni JW, Seid M, Kurtin PS. PedsQL 4.0: reliability and validity of the Pediatric Quality of Life Inventory version 4.0 generic core scales in health and patient populations. Med Care. 2001;39:800-12.

24. Baltazar Cl. Adaptação e Validação para a cultura portuguesa do Pediatric Quality of Life Inventory TM - PedsQLTM 4.0 - Generic Core Scales. [Dissertação de Mestrado], Faculdade de Motricidade Humana da Universidade Técnica de Lisboa, 2008.

25. Spencer NJ, Coe C. The development and validation of a measure of parent-reported child health and morbidity: The Warwick Child Health and Morbidity Profile. Child Care Health Dev. 1996:22:367-79.

26. Apajasalo M, Sintonen H, Holmberg C, Sinkkonen J, Aalberg V, Pinko H, et al. Quality of life in early adolescence: a sixteen dimensional healthrelated measure (16D). Qual Life Res. 1996:5:205-11.

27. Apajasalo M, Rautonen J, Holmberg C, Sinkkonen J, Aalberg V, Pinko $\mathrm{H}$, et al. Quality of life in pre-adolescence: a 17-dimensional healthrelated measure (17D). Qual Life Res. 1996;5:532-8.

28. Phipps S, Hinds PS, Channell S, Bell GL. Measurement of behavioral, affective, and somatic responses to pediatric bone marrow transplantation: development of the BASES scale. J Pediatr Oncol Nurs. 1994;11:109-17.

29. Armstrong FD, Toledano SR, Miloslavich K, Lackman-Zeman L, Levy JD, Gay CL, et al. The Miami pediatric quality of life questionnaire: parent scale. Int J Cancer. 1999;12:321-8.

30. Varni JW, Burwinkle T, Katz ER, Meeske K, Dickinson P. The PedsQL in pediatric cancer: reliability and validity of the Pediatric Quality of Life Inventory Generic Core Scales, Multidimensional Fatigue Scale, and Cancer Module. Cancer. 2002;94:2090-106.

31. Goodwin DAJ, Boggs SR, Graham-Pole J. Development and validation of the pediatric oncology quality of life scale (POQOL). Psychol Assess. 1994;6:321-8.

32. Silva M, Ribeiro JLP. Pediatric Oncology Quality of Life ScalePOQOLS: adaptação de um instrumento para a população portuguesa. Psicol Saúde Doenças. 2008;9:131-41.

33. Lansky LL, List MA, Lansky SB, Cohen ME, Sinks LF. Toward the 
development of a play performance scale for children (PPSC). Cancer. 1995;56:1837-40.

34. Mulhern RK, Fairclough DL, Friedman AG, Leigh LD. Play performance scale as an index of quality of life of children with cancer. Psychol Assess: J Consult Clin Psychol. 1990;2:149-55.

35. Eiser $\mathrm{C}$, Morse R. A review of measure of quality of life for children with chronic illness. Arch Dis Child. 2001;84:205-11.

36. Eiser C, Morse R. Quality-of-life measures in chronic diseases of childhood. Health Technol Assess. 2001;5:1-162.

37. Hanks G, Cherny N, Christakis NA, Fallon M, Kaasa S, Portenoy R. Oxford Textbook of Palliative Medicine. $4 \mathrm{t}^{\mathrm{h}}$ ed. Oxford: Oxford University Press; 2010.

38. Connolly M, Johnson J. Measuring quality of life in pediatric patients Working Paper 98-1. Edmonton: Institute of Pharmaco-Economics; 1998. 


\section{Instrumentos de Medição da Qualidade de Vida em Idade Pediátrica em Cuidados Paliativos}

Acta Med Port 2015:28:501-512

Publicado pela Acta Médica Portuguesa, a Revista Científica da Ordem dos Médicos

Av. Almirante Gago Coutinho, 151

1749-084 Lisboa, Portugal.

Tel: +351218428 215

E-mail: submissao@actamedicaportuguesa.com

www.actamedicaportuguesa.com

ISSN:0870-399X | e-ISSN: 1646-0758

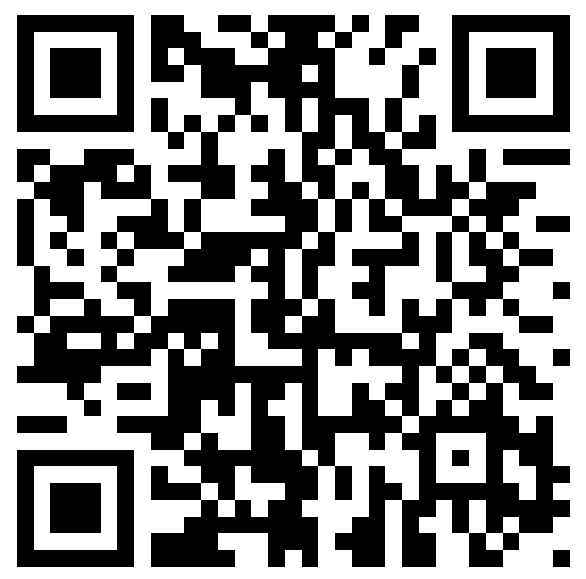

